\title{
Cтатьи
}

\section{ДИАГНОСТИКА УРОВНЯ РАЗВИТИЯ РЕГУЛЯТОРНЫХ ФУНКЦИЙ В СТАРШЕМ ДОШКОЛЬНОМ ВОЗРАСТЕ}

\author{
О.В. АЛМАЗОВА
}

${ }^{a}$ Московский государственный университет имени М.В. Ломоносова, 119991, Россия, Москва, Ленинские горь, 1

\section{Резюме}

В статье рассматривается модель регуляторных функций А. Мияке, примененная к дошкольному возрасту. Основной задачей исследования выступила апробация диагностического инструментария NEPSY-II для оценки уровня развития трех основных компонентов регуляторных функций (рабочей памяти, когнитивной гибкости и торможения) у российских дошкольников. Выборку исследования составили 267 детей (143 мальчика и 124 девочки) в возрасте 5-6 лет ( $\mathrm{Me}=5.6$ года, $\mathrm{SD}=0.3)$, посещающих старшую группу детских садов в г. Москве. В результате сравнения результатов американских и российских детей 5-6 лет выявлены некоторые кросс-культурные особенности: память на образы в среднем больше развита у российских, а на пространственные представления - у американских детей. Для проверки конвергентной валидности инструментария на части выборки $(\mathrm{N}=48)$, проведен корреляционный анализ результатов выполнения дошкольниками двух батарей методик - апробируемой и разработанной под руководством Л.А. Венгера. Обнаруженные взаимосвязи подтвердили возможность использования рассматриваемого инструментария для диагностики регуляторных функций. Анализ данных исследования показал, что уровни развития слухоречевой и зрительной рабочей памяти старших дошкольников не связаны между собой. Однако слухоречевая память во многом лежит в основе развития когнитивной гибкости внимания (переключения), а зрительная - сдерживающего контроля (торможения). На основании результатов диагностики выделены и описаны четыре различных варианта развития регуляторных функций в старшем дошкольном возрасте. Дети с достаточно высоким уровнем развития регуляторных функций распределены по двум группам в соответствии с ориентацией: на высокую скорость выполнения заданий и на высокое качество выполнения заданий (избегание ошибок).

Ключевые слова: дошкольный возраст, регуляторные функции, произвольность, слухоречевая память, зрительная память, гибкость внимания, сдерживающий контроль. 
Одним из самых важных достижений старшего дошкольного возраста и предиктором успешной адаптации и обучения в школе является развитие произвольности или регуляторных функций (Выготский, 1984; Эльконин, 2006; Смирнова, 2015; Blair, 2002; Welsch et al., 2010; Willoughby et al., 2012; Yeniad et al., 2013; и др.). Хотя между понятиями «произвольность» (в культурно-историческом подходе) и «регуляторные функции» (neuroscience) есть методологические различия, в основе лежат схожие процессы (Алмазова и др., 2016; Ахутина и др., 2014; Лурия, 2002).

Согласно модели регуляторных функций А. Мияке (Miyake et al., 2000), нейропсихологической основой для овладения собственным поведением является группа когнитивных навыков, обеспечивающих целенаправленное решение задач и адаптивное поведение в новых ситуациях, которые объединяются под общим названием «регуляторные функции» («executive functions»). Они обеспечивают мониторинг и контроль мышления и деятельности через смещение процессов в сторону стимула, связанного с выполнением задания, несмотря на вторичные задачи и помехи. В нейрокогнитивных исследованиях процесс торможения и рабочая память рассматриваются в качестве основных процессов, обеспечивающих произвольную регуляцию (Barkley, 2001; Solovieva, Quintanar, 2015; Verbitskaya et al., 2015).

Регуляторные функции делятся на следующие основные компоненты: рабочую память («working memory») - зрительную и слухоречевую, гибкость внимания или переключение («cognitive flexibility»), которое связано со способностью переходить от одного правила к другому, и сдерживающий контроль («inhibitory control»), который предполагает торможение доминирующего ответа в пользу требуемого в задании. Эти компоненты связаны друг с другом, но они могут рассматриваться и как самостоятельные, отдельно друг от друга, поэтому данная модель получила название «единство с разнообразием» («unity-with-diversity»). Несмотря на то что данная модель была изначально основана на результатах, полученных на взрослых, возможность ее использования при описании развития в детском возрасте была подтверждена в работах зарубежных исследователей (Diamond et al., 2002; Diamond, Lee, 2011; Lehto et al., 2003; Visu-Petra et al., 2012).

Регуляторные функции в предложенном выше понимании, как целостный конструкт, в отечественной психологии практически не исследовались (Веракса, Васильева, Арчакова, 2016). В связи с этим нами был подобран и апробирован на российской выборке диагностический инструментарий для оценки уровня развития регуляторных функций у старших дошкольников (5-6 лет).

\section{Методики}

Для диагностики развития регуляторных функций был использован набор из четырех методик, широко применяющихся в зарубежной практике. Бо́льшую часть использованных в исследовании методов составляют субтесты нейропсихологического диагностического комплекса NEPSY-II 
(Korkman et al., 2007), направленного на оценку психического развития детей в возрасте 3-16 лет.

Уровень развития рабочей памяти измерялся двумя методиками:

1. Субтест «Повторение предложений» (Sentences Repetition, NEPSY-II) слухоречевая память;

2. Субтест «Память на конструирование» (Memory for Designs, NEPSY-II) зрительная память.

Переключение и торможение диагностировались при помощи следующих методик:

1. Сортировка карт по изменяемому признаку (Dimensional Change Card Sort (DCCS)) (Zelazo, 2006);

2. Субтест «Торможение» (Inhibition, NEPSY-II).

Кроме того, для отсеивания детей с возможными нарушениями интеллектуального развития использовались Цветные прогрессивные матрицы Дж. Равена (Raven et al., 1998).

Так как все методики, кроме Цветных прогрессивных матриц Дж. Равена, не являются широко распространенными в отечественной психологии, опишем их подробнее.

В субтесте Повторение предложений используются 17 предложений. Предложения в методике организованы таким образом, что происходит постепенное усложнение стимульного материала (предложения становятся и длиннее, и сложнее по структуре). Так, первое предложение для повторения состоит из двух слов - «Спокойной ночи», а двенадцатое - «Женщина, стоящая рядом с человеком в зеленом пиджаке, - моя тетя». Опущение слова, замена или добавление слова считается ошибкой. Изменения в порядке слов, перемещенное слово также считается ошибкой. За каждое предложение ребенку начисляется 2 балла в случае отсутствия ошибок, 1 балл - за 1 или 2 ошибки, 0 баллов - за 3 и более ошибок или при отсутствии ответа от ребенка. Если за 4 предложения подряд ребенок получал 0 баллов, тестирование прекращалось. Исходный материал (предложения из оригинала методики) был переведен профессиональным переводчиком вначале с английского языка на русский, потом другим переводчиком был сделан обратный перевод. После чего был сделан анализ расхождений и проведена корректировка.

При помощи субтеста Память на конструирование происходит измерение сразу двух аспектов зрительной памяти - памяти на «образы» («Content score» - выбор картинок, как в предъявленном образце, из массива похожих) и пространственного расположения карточек на поле («Spatial score» - выбор места, где были расположены карточки в образце). По каждому заданию начисляется по одному баллу за каждую правильно выбранную карточку, по одному - за каждое правильно указанное место, занятое в эталоне, и по два бонусных балла - за каждое полное соответствие эталону (правильно выбранная карточка, помещенная в нужное место). Таким образом, результатом 
выполнения методики являются несколько оценок - оценки памяти: 1) на образы, 2) на пространственное расположение; 3) на сочетание первого и второго.

Методика Сортировка карт по изменяемому признаку содержит три серии заданий. В первой ребенок сортирует предложенные ему 6 карточек по цвету (красные откладываются в одну сторону, синие - в другую). Во второй сортировка происходит «по форме» 6 карточек (лодочки откладываются в одну сторону, зайцы - в другую). В третьем задании ребенок должен, ориентируясь на внешний для цвета или формы стимул - наличие или отсутствие черной рамки на карточке, раскладывать 12 карточек либо исходя из формы, либо исходя из цвета объекта на картинке. В каждой серии, за каждый правильный ответ начисляется по 1 баллу. Переключение с задания первой серии на задания второй является показательным для оценки уровня развития переключения у детей 3-4 лет. К 5-6 годам с этим справляются абсолютное большинство дошкольников. Таким образом, хотя мы оцениваем количество правильно разложенных карточек в каждой серии, решающим для определения уровня развития переключения в старшем дошкольном возрасте является балл, набранный ребенком в третьей серии.

Методика Торможение состоит из двух частей - называние и собственно торможение. Предъявляется серия из 40 геометрических фигур (квадратов и кругов). На первом этапе ребенка просят назвать все фигуры в том порядке, в котором они находятся на карточке. На втором этапе правила меняются: когда ребенок видит «круг», он должен сказать «квадрат», а когда видит «квадрат» - «круг». Для каждой части предусмотрена тренировка. Фиксируются время, затраченное на выполнение задания, количество допущенных ошибок и количество самоисправлений.

\section{Выборка и процедура исследования}

Выборку исследования составили 267 детей в возрасте 5-6 лет (Mе = 5.6 года, $\mathrm{SD}=0.3)$, посещающих старшую группу детских садов в г. Москве, из них 143 мальчика и 124 девочки. Данное исследование было проведено в 2016 учебном году. Обследования проводились в индивидуальном порядке, в тихом помещении. Все задания предъявлялись ребенку в игровой форме, что обеспечивало высокую степень включенности ребенка в их выполнение. С каждым ребенком было проведено по две встречи продолжительностью 15-20 минут каждая.

Авторы диагностического комплекса NEPSY-II выделяют следующие ограничения для детей, которые участвовали в исследовании при стандартизации комплекса.

Ребенок исключался из исследования, если:

1) проходил любое другое нейропсихологическое исследование в течение 6 месяцев;

2) имеет слуховые или зрительные нарушения или является дальтоником;

3) имеет психиатрический диагноз, нейропсихологические нарушения, дефицит внимания, гиперактивность, задержку интеллектуального развития; 
4) родился с очень маленьким весом - менее 2.5 кг;

5) на момент исследования принимает медицинские препараты, способные повлиять на результаты (стимуляторы, антидепрессанты, лекарства от тревожности и т.д.);

6) в истории развития зафиксированы потеря сознания более чем на 5 минут, впадание в кому (по шкале Глазго до 14) и т.д.;

7) имеет языковые трудности или язык исследования не родной для ребенка (языковые трудности не включают в себя проблему с артикуляцией, если это не затрудняет коммуникацию с ребенком) (Korkman et al., 2007).

Из этих условий мы не контролировали 4, 5 и 6. Уровень интеллектуального развития проверялся при помощи Цветных прогрессивных матриц Дж. Равена.

\section{Описание результатов}

\section{Общий анализ данных}

При помощи Цветных прогрессивных матриц Дж. Равена из исследования были исключены дошкольники с подозрениями на нарушения познавательного развития (3 дошкольника).

В таблице 1 представлены основные психометрические характеристики результатов выполнения методик на оценку развития разных компонентов регуляторных функций, полученные в нашем исследовании и при адаптации комплекса методик NEPSY-II (нормы США).

Из таблицы 1 можно выделить общие тенденции и особенности выполнения методик в различных выборках:

1) Небольшая разница в средних баллах по методике «Повторение предложений» объясняется, на наш взгляд, не разницей в уровне развития слухоречевой памяти, а небольшим различием в сложности структур предложений в русской и англоязычной версии.

1) «Память на конструирование». Средние баллы за воспроизведение образа выше у российских, а за воспроизведение места - у американских детей. При этом общий балл в среднем выше у российских дошкольников. Сами картинки в среднем точнее запоминаются российскими дошкольниками, а расположение в пространстве - их американскими сверстниками, что, скорее всего, объясняется различием образовательных программ. Большое же различие в средних общих баллах свидетельствует о том, что российские дети чаще выполняли задание полностью хорошо, т.е. воспроизводили и правильно выбранные объекты, и их место на поле, что позволяло получать максимально возможные бонусные баллы.

2) Хотя данные по основным психометрическим характеристиками методики «Сортировка карт по изменяемому признаку» не представлены, но данные большинства исследований автора методики показывают, что к шести годам около половины детей начинают успешно справляться с последней 
Таблица 1

Основные психометрические характеристики оценок компонентов регуляторных функций у российских и американских дошкольников

\begin{tabular}{|l|c|c|c|c|}
\hline \multirow{2}{*}{\multicolumn{2}{|c|}{ Компоненты РФ }} & \multicolumn{4}{c|}{ Страна } \\
\cline { 2 - 5 } & \multicolumn{2}{|c|}{ Россия } & \multicolumn{2}{c|}{ США } \\
\cline { 2 - 5 } & $\mathrm{Me}$ & $\mathrm{SD}$ & $\mathrm{Me}$ & $\mathrm{SD}$ \\
\hline Память на конструирование, образ (MDC) & 39.5 & 6.2 & 31.5 & 6.1 \\
\hline Память на конструирование, расположение (MDS) & 16.6 & 6.7 & 19.2 & 6.3 \\
\hline Память на конструирование, общий балл (MDT) & 79.9 & 23.4 & 63.4 & 22.6 \\
\hline Повторение предложений (SR) & 19.6 & 4.3 & 17.2 & 5.4 \\
\hline Сортировка по цвету (DCCS_Pr) & 5.9 & 0.3 & & \\
\hline Сортировка по форме (DCCS_Рs) & 5.5 & 1.2 & & \\
\hline Сортировка с рамкой (DCCS_В) & 7.5 & 2.9 & & \\
\hline Сортировка, общий балл (DCCS_Т) & 18.9 & 3.5 & & \\
\hline Называние, время (INT) & 44 & 11 & & \\
\hline Торможение, неисправленные ошибки (IIUE) & 3.1 & 5.3 & & \\
\hline Торможение, исправленные ошибки (IICE) & 1.9 & 1.6 & & \\
\hline Торможение, время (IIT) & 61 & 15 & & \\
\hline
\end{tabular}

пробой методики (набирают 9 или более баллов из 12). Среди наших респондентов $38 \%$ дошкольников справились с этим блоком. Таким образом, можно говорить о близости результатов по этой методике.

Вернемся к результатам нашего исследования. Были проверены гендерные различия в результатах выполнения всех методик (использован критерий Манна-Уитни для двух независимых выборок). Получено, что девочки значимо лучше выполняют задания по сортировке карт по форме ( $U=7906.0$; $p=0.001)$ и делают значимо меньше ошибок (как исправленных, так и неисправленных) в пробе на торможение $(U=7103.5 ; p<0.000$ и $U=7859.5$; $p=0.020)$.

В таблице 2 представлены результаты корреляционного анализа данных, полученных по разным методикам комплекса (был использован коэффициент корреляции Спирмена).

Прежде всего остановимся на связях результатов выполнения заданий, направленных на диагностику уровня развития разных компонентов регуляторных функций.

Запоминание образа при выполнении методики на проверку зрительной памяти оказалось связанным со временем, которое требовалось для выполнения двух проб в методике «Торможение», т.е. чем лучше запоминается образ, тем быстрее происходит работа с называнием фигуры и ее инверсией. 
О.В. Алмазова, Д.А. Бухаленкова, А.Н. Веракса

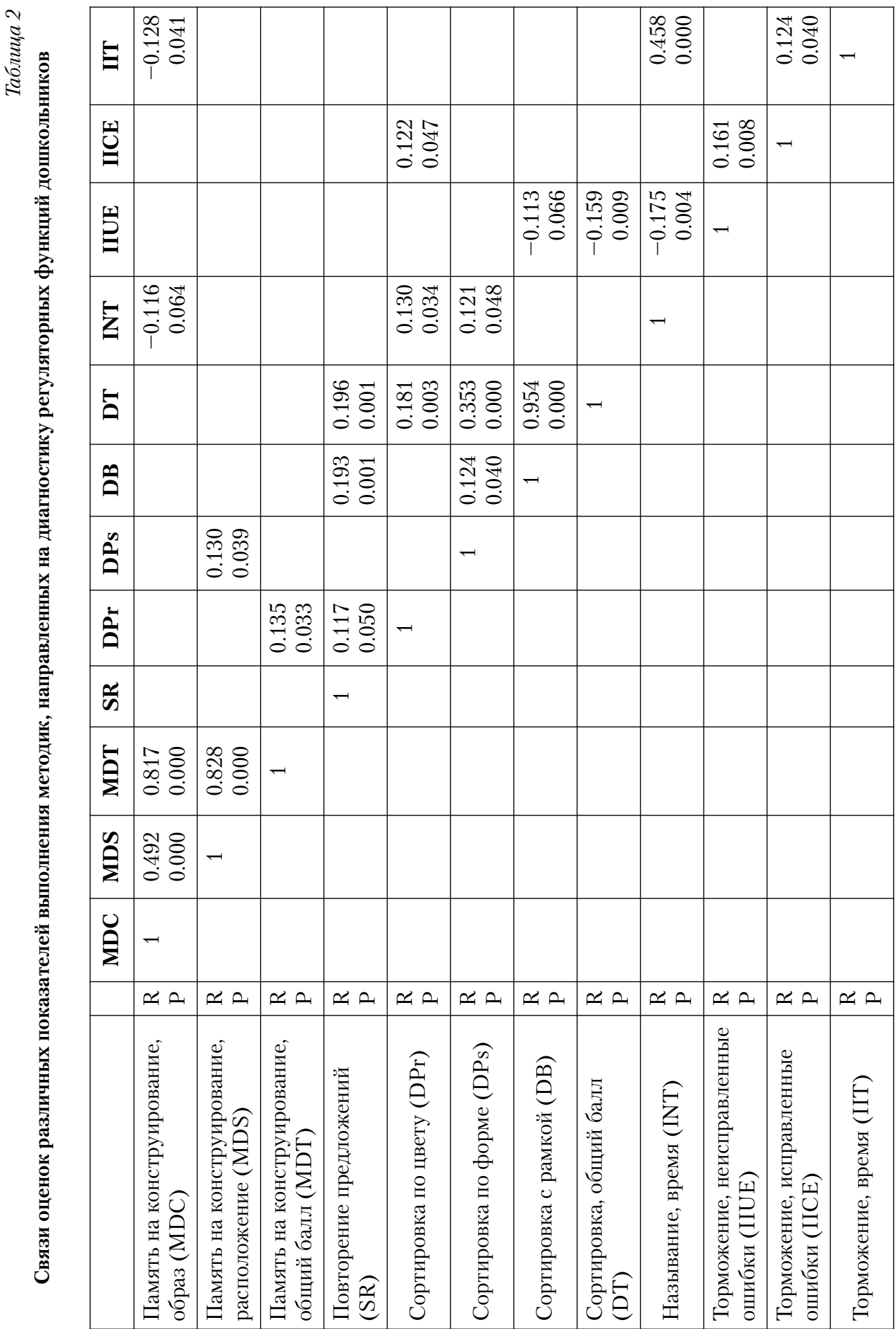


Результаты, полученные при выполнении методики «Повторение предложений», связаны с разными параметрами методики «Сортировка карт по изменяемому признаку», что может, к примеру, свидетельствовать о том, что хорошо развитая слухоречевая память способствует лучшему усвоению инструкций.

Что касается результатов методик на торможение и переключение, то количество неисправленных ошибок связано (обратно) с успешностью выполнения третьего задания на переключение (сортировка по изменяемому признаку), а время называния - с успешностью выполнения первых двух проб на переключение.

Оценки же внутри методик оказались связанными между собой, что стало ожидаемым результатом.

\section{Конвергентная и конструктная валидность инструментария}

Оценка конвергентной валидности проводилась путем вычисления на части выборки ( $\mathrm{N}=48,24$ девочки и 24 мальчика) корреляции результатов, полученных по описанным выше методикам, с результатами выполнения дошкольниками батареи методик, разработанных в лаборатории Л.А. Венгера (Венгер и др., 1986). Причинами использования этого инструментария являются его широкая распространенность в России, использование культурно-исторического подхода Л.С. Выготского как основы построения методологии методического инструментария и особое внимание к развитию произвольности через освоение средств наглядного моделирования (Там же).

Были введены в рассмотрение следующие методики:

1. Методика Эталоны (Венгер, Холмовская, 1978) позволяет определить развитие уровня восприятия у детей старшего дошкольного возраста, а именно умение соотносить сенсорные эталоны формы с реальными объектами.

2. Методика Систематизация (Там же) показывает степень сформированности у дошкольника таких действий логического мышления, как сериация и классификация.

3. Методика Схематизация (Там же) отражает уровень овладения ребенком действий наглядного моделирования: при выполнении заданий ребенок использует условно-схематическое изображение в качестве образца или правила, для достижения положительного результата ему необходимо строго следовать заданной последовательности ориентиров. Таким образом, при выполнении данного задания задействованы процессы восприятия, нагляднообразного мышления, произвольного внимания и регуляции своей деятельности.

4. Методика Самое непохожее (Венгер и др., 1995) позволяет изучить развитие восприятия и мышления детей, оценить уровень овладения такими мыслительными операциями, как анализ, сравнение и обобщение признаков.

5. Методики запоминания 10 слов (Там же) и 10 предметов (Марцинковская, 2000) позволяют определить уровень развития рабочей слуховой и зрительной памяти. 
Проведенное сравнительное исследование показало, что результаты проведения двух диагностических наборов достаточно хорошо соотносятся друг с другом и позволяют увидеть вклад уровня развития, памяти и произвольного внимания в успешность выполнения заданий, направленных на изучение регуляторных функций. Например, количество ошибок, которое допускает ребенок при выполнении пробы на называние методики Торможение, оказалось связано (обратно) с результатами большинства методик, направленных на диагностику степени усвоения ребенком сенсорных эталонов и уровня развития наглядно-образного мышления (Эталоны $(r=-0.359, p=0.015)$, Систематизация $(r=-0.315, p=0.042)$, Схематизация $(r=-0.342, p=0.022))$. А это, в свою очередь, и свидетельствует о связи представлений о сдерживающем контроле как компоненте регуляторных функций и произвольности мыслительных процессов, согласно теории способностей Л.А. Венгера.

Связь же результатов методик Самое непохожее и Сортировка карт по изменяемому признаку $(r=0.402, p=0.006)$ может указывать на то, что в основе выполнения и той и другой методики лежит умение ориентироваться на свойства внешнего эталона для совершенствования своих действий.

Результаты методики, направленные на диагностику уровня развития слухоречевой памяти 10 слов и Повторение предложении, оказались связаны $(r=0.328 ; p=0.028)$, тогда как результаты методик 10 предметов и Память на конструирование оказались связаны частично: результаты методики 10 предметов связаны только с одним из аспектов, рассматриваемых в методике Память на конструирование, а именно с памятью на образы $(r=0.402, p=0.006)$. Это позволило нам сделать вывод, что Память на конструирование дает больше диагностических данных, чем 10 предметов.

Таким образом, мы может говорить о диагностике произвольности с помощью данных методов, отмечая высокую детализацию развития различных аспектов регуляторных функций с использованием методик, разработанных в зарубежных подходах.

Для проверки того, что в методике Повторение предложений происходит постепенное усложнение заданий, было проведено сравнение результатов, полученных в каждом из вопросов. Итогом этой работы стало перемещение некоторых предложений для дальнейшей работы с методикой. Так, к примеру, предложение 14 «Подростки в нашем доме собирают деньги на строительство развлекательного центра» оказалось значимо более трудным для дошкольников, чем предложение 15 «Мы сложили еду для пикника обратно в корзину, потому что приближалась буря» (критерий Вилкоксона для двух зависимых выборок, $\mathrm{W}=-5.808, p<0.000)$. В связи с этим на следующем этапе стандартизации порядок предъявления предложений будет несколько изменен.

\section{Типология развития регуляторных функций в старшем дошкольном возрасте}

Достаточно большой разброс данных в результатах выполнения методик на диагностику уровня развития регуляторных функций (таблица 1) позволил 
выдвинуть предположение о возможности построения типологии развития регуляторных функций дошкольников 5-6 лет. При помощи кластерного анализа (метод k-средних) данных всех рассматриваемых методик респонденты были распределены на четыре группы (количество групп было выведено опытным путем). В таблице 3 представлены центры полученных кластеровтипов (средневзвешенные значения).

При помощи критерия Краскела-Уоллиса для нескольких независимых выборок проверили, являются ли различия между оценками дошкольников разных групп значимыми. Значимые различия наблюдаются по результатам всех методик, кроме «Сортировка по изменяемому признаку». Отметим, что именно по этой методике относительный разброс данных наименьший. Опишем получившиеся группы дошкольников подробнее.

1 тип (12\% выборки). Дошкольники, попавшие в эту группу, имеют невысокие оценки развития зрительной памяти, тратят много времени при выполнении проб на называние и торможение, допускаюя при этом довольно много ошибок, которые не исправляются. Слухоречевая память развита хорошо.

2 тип (41\% выборки). Респонденты этой группы имеют невысокие оценки развития зрительной памяти, быстро выполняют пробы на называние и торможение. При этом в пробе на торможение допускается много ошибок, бо́льшая часть которых не исправляется. Слухоречевая память у дошкольников этой группы развита хуже, чем у остальных дошкольников.

3 тип (18\% выборки). Дошкольники из этой группы имеют высокие оценки развития зрительной памяти. Затраты времени на выполнение проб на называние и торможение средние. При этом допускается минимальное число оши-

Таблица 3

Центры кластеров по всем оценкам компонент регуляторных функций

\begin{tabular}{|l|c|c|c|c|}
\hline & $\mathbf{1}$ тип & $\mathbf{2}$ тип & $\mathbf{3}$ тип & $\mathbf{4}$ тип \\
\hline Память на конструирование, образ (MDC) & 35.89 & 35.98 & 42.55 & 44.11 \\
\hline Память на конструирование, расположение (MDS) & 13.32 & 12.76 & 21.11 & 21.34 \\
\hline Память на конструирование, общий балл (MDT) & 65 & 61 & 97 & 101 \\
\hline Повторение предложений (SR) & 21 & 19 & 20 & 21 \\
\hline Сортировка по цвету (DCCS_Pr) & 6 & 6 & 6 & 6 \\
\hline Сортировка по форме (DCCS_Ps) & 5 & 5 & 6 & 6 \\
\hline Сортировка с рамкой (DCCS_B) & 8 & 7 & 7 & 7 \\
\hline Сортировка, общий балл (DCCS_T) & 19 & 19 & 19 & 19 \\
\hline Называние, время (INT) & 65.5 & 41.4 & 49.6 & 37.8 \\
\hline Торможение, неисправленные ошибки (IIUE) & 5.2 & 3.6 & 1.9 & 3.4 \\
\hline Торможение, исправленные ошибки (IICE) & 1.9 & 1.9 & 2.4 & 1.8 \\
\hline Торможение, время (IIT) & 94.1 & 57.6 & 70.6 & 51.0 \\
\hline
\end{tabular}


бок, бо́льшая часть которых исправляется. Развитие слухоречевой памяти находится на среднем уровне.

4 тип (29\% выборки). Респонденты, попавшие в эту группу, получили самые высокие оценки при тестировании развития зрительной памяти. Слухоречевое развитие также находится на высоком уровне. Затраты времени на выполнение проб на называние и торможение - минимальные по сравнению с другими группами. При этом ошибки в пробе на торможение (количество ошибок - на среднем уровне) чаще не исправляются, чем исправляются.

Важными представляются различия между третьим и четвертым типом развития регуляторных функций дошкольников. Дошкольники третьего типа предпочитают тратить больше времени, стараясь избегать ошибок, тогда как дети четвертого типа настроены на быстрое выполнение задания, не так сильно заботясь о точности получаемого результата.

\section{Обсуждение результатов и выводы}

В работе представлен первый этап адаптации и стандартизации комплекса методик, направленных на диагностику уровня развития регуляторных функций у старших дошкольников и на изучение основных их компонентов.

Проведен сравнительный анализ результатов, полученных на российских и американских дошкольниках. Выявлены как общие, так и различные черты, которые можно объяснить разницей в системах образования и опыта ребенка. Так, к примеру, уровень развития памяти на образы (картинки) оказался выше у российских старших дошкольников, а памяти на пространственные представления - у их американских сверстников. Мы находим полученные результаты логичными, так как в американском начальном образовании обучение по большей части ведется путем освоения детьми практических навыков, что и ведет к лучшей ориентации в пространстве. В российской школе занятия проводятся чаще всего за партами и включают теоретическую часть, а это, в свою очередь, помогает больше сосредотачиваться на деталях изображений (Борисова, 2010).

В результате проверки связей между результатами выполнения разных методик было получено, что уровни развития слухоречевой и зрительной памяти не связаны между собой. При этом слухоречевая память в большей степени обеспечивает успешность выполнения задач на переключение, а зрительная - на торможение. Уровни же развития переключения и торможения оказались связанными.

Для проверки конвергентной валидности методического комплекса были сравнены (на части выборки) результаты выполнения дошкольниками двух батарей методик: заявленной в исследовании и разработанной в лаборатории Л.А. Венгера. Большое количество связей подтверждает правомерность использования выбранного комплекса для диагностики уровня развития регуляторных функций.

Для получения более объемной картины вариантов развития регуляторных функций старших дошкольников респонденты были разделены на четыре 
группы. Были описаны особенности и численный состав каждой группы. При достаточно высоком уровне развития регуляторных функций можно увидеть начало формирования двух основных стратегий выполнения задания: первая направлена на как можно более быстрое выполнение, вторая - на безошибочHое.

\section{Заключение}

Уже на данном этапе можно видеть диагностические возможности предложенного к рассмотрению инструментария, направленного на диагностику уровня развития регуляторных функций.

На следующем этапе с внесением небольших изменений в материалы, с применением более строгого контроля возраста детей на момент проведения каждой из методик будут разработаны нормы для российской выборки. Кроме того, планируется проведение ретеста на аналогичных материалах и контроль таких переменных, как личность тестера, географическое расположение детских садов и т.д.

\section{Литература}

Алмазова, О. В., Бухаленкова, Д. А., Веракса, А. Н. (2016). Произвольность в дошкольном возрасте: сравнительный анализ различных подходов и диагностического инструментария. Национальный психологческий журнал, 24(4), 14-22.

Ахутина, Т. В., Варако, Н. А., Григорьева, В. Н., Микадзе, Ю. В., Скворцов, А. А., Фуфаева, Е. В. (2014). Нейропсихологическая диагностика и нейропсихологическал реабилитачия нарушений управляющих (регуляторных) функций и критичности при повреждениях головного мозга. Клинические рекомендащии. М.: Союз реабилитологов России.

Борисова, С. П. (2010). Современное дошкольное образование за рубежом. Теория и практика общественного развития, 3, 132-137.

Венгер, Л. А., Агаева, Е. Л., Венгер, Н. Б., Говорова, Р. И., Дьяченко, О.М., Журова Л.Е., ... Цеханская, Л. И. (1986). Развитие познавательных способностей в процессе дошкольного воспитания. М.: Педагогика.

Венгер, Л. А., Агаева, Е. Л., Бардина, Р. И., Брофман, В. В., Булычева, А. И., Бурлакова, И. А., ... Цеханская, Л. И. (1995). Психолог в детском саду. М.: ИНТОР.

Венгер, Л. А., Холмовская, В. В. (1978). Диагностика умственного развития дошкольников. М.: Педагогика.

Веракса, А. Н., Васильева, М. Д., Арчакова, Т. О. (2016). Развитие произвольности регуляторных функций в дошкольном возрасте: обзор современных зарубежных исследований. Вопросы психологии, 6, 150-166.

Веракса, А. Н., Якупова, В. А., Алмазова, О. В., Мартыненко, М. Н. (2016). Познавательное и социальное развитие дошкольников в контексте готовности к школе. Вестник СанктПетербургского университета. Серия 16. Психология и педагогика, 3, 97-108.

Выготский, Л. С. (1984). Детская психология. В кн. Л. С. Выготский, Собрание сочинений (т. 4, с. 243-385). М.: Педагогика. 
Лурия, А. Р. (2002). Основы нейропсихологии. М.: Академия.

Марцинковская, Т. Д. (2000). Детская практическая психология. М.: Гардарика.

Смирнова, Е. О. (2015). К проблеме воли и произвольности в культурно-исторической психологии. Культурно-историческая психология, 11(3), 9-15.

Эльконин, Д. Б. (2006). Детская психология. М.: Академия.

Ссылки на зарубежные источники см. в разделе References после англоязычного блока.

Алмазова Ольга Викторовна - доцент, кафедра возрастной психологии, факультет психологии, ФГБОУ ВО МГУ имени М.В. Ломоносова, кандидат психологических наук.

Сфера научных интересов: привязанность, эмоциональная, когнитивная и регуляторная сферы развития ребенка в дошкольном и младшем школьном возрасте.

Контакты: almaz.arg@gmail.com

Бухаленкова Дарья Алексеевна - младший научный сотрудник, кафедра психологии образования и педагогики, факультет психологии, ФГБОУ ВО МГУ имени М.В. Ломоносова, кандидат психологических наук.

Сфера научных интересов: эмоциональная, когнитивная и регуляторная сферы развития ребенка в дошкольном и младшем школьном возрасте.

Контакты: d.bukhalenkova@inbox.ru

Веракса Александр Николаевич - заведующий кафедрой, профессор, кафедра психологии образования и педагогики, факультет психологии, ФГБОУ ВО МГУ имени М.В. Ломоносова, доктор психологических наук, профессор, член-корреспондент РАО.

Сфера научных интересов: эмоциональная, когнитивная и регуляторная сферы развития ребенка в дошкольном и младшем школьном возрасте.

Контакты: veraksa@yandex.ru 


\title{
Assessment of the Level of Development of Executive Functions in the Senior Preschool Age
}

\author{
O.V. Almazova ${ }^{a}$, D.A. Bukhalenkova ${ }^{a}$, A.N. Veraksa ${ }^{a}$ \\ ${ }^{a}$ Lomonosov Moscow State University, 1 Leninskie Gory, Moscow, 119991, Russian Federation
}

\begin{abstract}
The article employs the model of executive functions by A. Miyake to preschool age. The main objective of the research was the approbation of the diagnostic instrument NEPSY-II to assess the level of development of the three main components of executive functions (working memory, cognitive flexibility and inhibition) in the Russian preschoolers. The sample consisted of 267 children (143 boys and 124 girls) aged $5-6(\mathrm{M}=5.6, \mathrm{SD}=0.3)$, who frequented the senior group of Moscow nursery schools. In the result of comparison of the performance of American and Russian children aged 5-6 years, certain cross-cultural differences were found: remembering images in general is more developed in Russian children, while spatial memory is better in American kids. To verify the convergent validity of the instrument on a partial sample $(\mathrm{N}=48)$, correlation analysis was conducted of the results of two batteries of tests: one that was approbated and one by L.A. Venger. The found correlations confirmed the feasibility to use this instrument for the executive functions assessment. The data analysis showed that the levels of audioverbal and visual working memory in senior preschoolers are not connected. But audio-verbal working memory is at large the foundation of the development of cognitive flexibility (switching), while visual working memory underlies the inhibition. Based on the results of the assessment four variants of executive functions in senior preschool age were distinguished and described. Children with relatively high level of development of executive functions were arranged in two groups in accordance with orientation: those who sought to perform the tasks faster and those who were focused on quality (avoidance of mistakes).
\end{abstract}

Keywords: preschool age, executive functions, voluntariness, audio-verbal working memory, visual working memory, cognitive flexibility, inhibition.

\section{References}

Akhutina, T. V., Varako, N. A., Grigorieva, V. N., Mikadze, Yu. V., Skvortsov, A. A., \& Fufaeva, E. V. (2014). Neiropsikhologicheskaya diagnostika i neiropsikhologicheskaya reabilitatsiya narusheniy upravlyayushchikh (regulyatornykh) funktsiy $i$ kritichnosti pri povrezhdeniyakh golovnogo mozga. Klinicheskie rekomendatsii [Neuropsychological diagnostics and neuropsychological rehabilitation of impairment of executive functions and criticism in brain injuries]. Moscow: Soyuz reabilitologov Rossii. (in Russian)

Almazova, O. V., Bukhalenkova, D. A., \& Veraksa, A. N. (2016). The voluntariness in the preschool age: a comparative analysis of various approaches and diagnostic tools. Natsionalny Psikhologichesky Zhurnal [National Psychological Journal], 24(4), 14-22. (in Russian) 
Barkley, R. A. (2001). The executive functions and self-regulation: An evolutionary neuropsychological perspective. Neuropsychology Review, 11(1), 1-29.

Blair, C. (2002). School readiness: Integrating cognition and emotion in a neurobiological conceptualization of children's functioning at school entry. American Psychologist, 57, 111-127.

Borisova, S. P. (2010). Sovremennoe doshkol'noe obrazovanie za rubezhom [Modern preschool education abroad]. Teoriya i Praktika Obshchestvennogo Razvitiya, 3, 132-137.

Diamond, A., \& Lee, K. (2011). Interventions shown to aid executive function development in children 4-12 years old. Science, 333, 959-964.

Diamond, A., Kirkham, N., \& Amso, D. (2002). Conditions under which young children can hold two rules in mind and inhibit a prepotent response. Developmental Psychology, 38, 352-362.

Elkonin, D. B. (2006). Detskaya psikhologiya [Child psychology]. Moscow: Akademiya.

Korkman, M., Kirk, U., \& Kemp, S. L. (2007). NEPSY II. Administrative manual. San Antonio, TX: Psychological Corporation.

Lehto, J., Juujarvi, P., Kooistra, L., \& Pulkkinen, L. (2003). Dimensions of executive functioning: evidence from children. The British Journal of Developmental Psychology, 21, 59-80.

Luria, A. R. (2002). Osnovy neiropsikhologii [Foundations of neuropsychology]. Moscow: Akademiya. (in Russian)

Martsinkovskaya, T. D. (2000). Detskaya prakticheskaya psikhologiya [Practical child psychology]. Moscow: Gardarika. (in Russian)

Miyake, A., Friedman, N. P., Emerson, M. J., Witzki, A. H., Howerter, A., \& Wager, T. (2000). The unity and diversity of executive functions and their contributions to complex "frontal lobe" tasks: A latent variable analysis. Cognitive Psychology, 41, 49-100.

Raven, J., Raven, J. C., \& Court, J. H. (1998). Manual for Raven's progressive matrices and vocabulary scales. Section 2: The coloured progressive matrices. Oxford, UK: Oxford Psychologists Press.

Smirnova, E. O. (2015). On the problem of will and self-regulation in cultural-historical psychology. Kul'turno-Istoricheskaya Psikhologiya [Cultural-Historical Psychology], 11(3), 9-15. (in Russian)

Solovieva, Yu., \& Quintanar, L. (2015). Qualitative syndrome analysis by neuropsychological assessment in preschoolers with attention deficit disorder with hyperactivity. Psychology in Russia: State of the Art, 8(3), 112-124.

Venger, L. A., Agaeva, E. L., Bardina, R. I., Brofman, V. V., Bulycheva, A. I., Burlakova, I. A., ... Tsekhanskaya, L. I. (1995). Psikholog $v$ detskom sadu [Psychologist in the nursery school]. Moscow: INTOR. (in Russian)

Venger, L. A., Agaeva, E. L., Venger, N. B., Govorova, R. I., D`yachenko, O.M., Zhurova L.E., ... Cexanskaya, L. I. (1986). Razvitie poznavatel’ny x sposobnostej v processe doshkol’nogo vospitaniya [Development of cognitive abilities in the process of preschool education]. Moscow: Pedagogika. (in Russian)

Venger, L. A., \& Kholmovskaya,V. V. (1978). Diagnostika umstvennogo razvitiya doshkol'nikov [Diagnostics of the cognitive development]. Moscow: Pedagogika. (in Russian)

Veraksa, A. N., Vasilieva, M. D., \& Archakova, T. O. (2016). Development of arbitrary regulatory functions in preschool children: A review of contemporary foreign studies. Voprosy Psikhologii, 6, 150166. (in Russian)

Veraksa, A. N., Yakupova, V. A., Almazova, O. V., \& Martynenko, M. N. (2016). Cognitive and social development of preschoolers in the context of school readiness. Vestnik of Saint Petersburg University. Series 16. Psychology. Education, 3, 97-108. (in Russian) 
Verbitskaya, L. A., Malykh, S. B., Zinchenko, Yu. P., \& Tikhomirova, T. N. (2015). Cognitive predictors of success in learning Russian. Psychology in Russia: State of the Art, 8(4), 91-100.

Visu-Petra, L., Cheie, L., Benga, O., \& Miclea, M. (2012). The structure of executive functions in preschoolers: An investigation using the NEPSY battery. Procedia - Social and Behavioral Sciences, 33, 627-631.

Vygotsky, L. S. (1984). Detskaya psikhologiya [Child psychology]. In L. S. Vygotsky, Sobranie sochineniy [Collected works] (Vol. 4, pp. 243-385). Moscow: Pedagogika. (in Russian)

Welsch, J. A., Nix, R. L., Blair, C., Bierman, K. L., \& Nelson, K. E. (2010). The development of cognitive skills and gains in academic school readiness for children from low-income families. Journal of Educational Psychology, 102, 43-53.

Willoughby, M. T., Kupersmidt, J. B, \& Voegler-Lee, M. E. (2012). Is preschool executive function causally related to academic achievement? Child Neuropsychology: A Journal on Normal and Abnormal Development in Childhood and Adolescence, 18(1), 79-91.

Yeniad, N., Malda, M., Mesman, J., Van IJzendoorn, M. H., \& Pieper, S. (2013). Shifting ability predicts math and reading performance in children: A meta-analytical study. Learning and Individual Differences, 23, 1-9. doi:10.1016/j.lindif.2012.10.004

Zelazo, P. D. (2006). The Dimensional Change Card Sort (DCCS): a method of assessing executive function in children. National Protocols, 1, 297-301.

Olga V. Almazova - associate professor, Department of Developmental Psychology, Faculty of Psychology, Lomonosov Moscow State University, Ph.D.

Research area: emotional, cognitive and regulatory spheres of preschoolers and primary schoolers. E-mail: almaz.arg@gmail.com

Daria A. Bukhalenkova - junior research fellow, Department of Psychology of Education and Pedagogics, Faculty of Psychology, Lomonosov Moscow State University, Ph.D.

Research area: emotional, cognitive and regulatory spheres of preschoolers and primary schoolers. E-mail: d.bukhalenkova@inbox.ru

Aleksandr N. Veraksa - head of the department, professor, Department of Psychology of Education and Pedagogics, Faculty of Psychology, Lomonosov Moscow State University, Ph.D. Research area: emotional, cognitive and regulatory spheres of preschoolers and primary schoolers. E-mail: veraksa@yandex.ru 